International Journal of Automotive and Mechanical Engineering (IJAME)

ISSN: 2229-8649 (Print); ISSN: 2180-1606 (Online); Volume 7, pp. 830-839, January-June 2013

CUniversiti Malaysia Pahang

DOI: http://dx.doi.org/10.15282/ijame.7.2012.3.0068

\title{
TRACKING CONTROL OF AN UNDERACTUATED GANTRY CRANE USING AN OPTIMAL FEEDBACK CONTROLLER
}

\author{
Firooz Bakhtiari-Nejad ${ }^{1}$, Mostafa Nazemizadeh ${ }^{2}$ and Hanieh Arjmand ${ }^{1}$ \\ ${ }^{\mathbf{1}}$ Department of Mechanics, Amirkabir University of Technology, Iran \\ ${ }^{2}$ Department of Mechanics, Damavand Branch \\ Islamic Azad University, Damavand, Iran \\ Tell: ++98-912-7376322 \\ Email: mn.nazemizadeh@gmail.com
}

\begin{abstract}
Gantry cranes have attracted a great deal of interest in transportation and industrial applications. To increase the effectiveness of gantry cranes, the control of such systems is considered vital. This paper is concerned with tracking the control of an underactuated gantry crane using an optimal feedback controller. The optimal control strategy takes into account a performance index, including integrated time and absolute error criterion. To do this, nonlinear dynamic equations of the system are derived using Lagrange's Principle. The minimum tracking error of the trolley and the minimum oscillation of the hoisting line are assumed as design parameters, and the best gains of the feedback controller are achieved. Finally, some tracking simulations are performed which demonstrate the capability of the simple proposed method in the optimal tracking control of a gantry crane.
\end{abstract}

Key words: Tracking control; gantry crane; optimal feedback; ITAE.

\section{INTRODUCTION}

Cranes are exceedingly applied in the transportation and construction fields. A crane consists of a hoisting mechanism which is suspended from a point on the support mechanism. The support mechanism moves the suspension point around the crane workspace, while the hoisting mechanism lifts and lowers the payload to deposit the payload at a target point. Based on the support mechanism, cranes can be classified as: gantry (overhead) cranes, tower (rotary) cranes, and boom crane. Gantry cranes are commonly composed of a trolley moving along a fixed support, while a hoisting mechanism (traditionally a cable and a hook) is suspended from a point on the trolley to transport the payload. Gantry cranes have attracted a great deal of interest in transportation and industrial applications, due to their low cost, ease of assembly, and low maintenance (Abdel-Rahman et al., 2003; Ahmad and Mohamed, 2009, 2010; Stahlbock and Vob, 2010). For the sake of minimum transportation time, high tracking accuracy, and minimum swing angle, the dynamic modelling and motion control of gantry crane systems have become appealing tasks in the field of control science. For the modelling of a crane two approaches are introduced in the literature survey: lumpedmass and distributed-mass models. In the distributed-mass procedure, the hoisting line is modelled as a string, and the payload is assumed as a lumped mass as a boundary condition of the system (Manning et al., 2010; Masoud, 2009; Rahn et al., 1999). On the 
other hand, the lumped-mass modelling of the crane considers a massless cable as the hoisting line, and a lumped mass as the payload. This approach has been widely employed in the modelling of gantry cranes (Ahmad, 2009; Dey et al., 2010; Kolonic et al., 2006; Zrnic et al., 1998). Furthermore, the dynamic motion of a crane can be exposed to some unwanted phenomena, such as inaccurate path travel, relatively large sway angles, and residual pendulations at the target point. These constraints degrade the efficiency of the crane's operation, and lead to time delays and higher operation costs. Thus the control of the dynamic motion of a gantry crane has recently received a great deal of interest. Hubbel et al. (1992) used an open-loop control method (named inputshaping method) to control the motion of a gantry crane. In this method, the input control profile was determined in such way that unwanted oscillations and residual pendulations were avoided during travel (Masoud and Daqaq, 2006). Solihin and Legowo (2010) proposed a Fuzzy-tuned PID controller for the anti-swing control of a crane. Moreover, a hybrid input-shaping strategy was implemented by Ahmad et al. (2009) to control a gantry crane system. However, although this method was effective, the input-shaping method changed from an open-loop control scheme, and was not robust to disturbances and parameter uncertainties (Dey et al., 2010). On the other hand, feedback control, which is well known to be more robust, is also adopted for the control of gantry crane systems. The first feedback control strategy for such a system was suggested by Hazlerigg (1972). A second order lead compensator was employed to dampen the payload pendulations. Moustafa and Ebied (1988) used a nonlinear modelling and anti-swing control method for an overhead crane. Yu et al. (1995) used a perturbation technique to separate the slow and fast dynamic behaviours of a gantry crane. Then they used a feedback controller (including two independent PD controllers) to track a pre-defined motion profile and suppress payload pendulations. Furthermore, a fuzzy logic feedback controller was proposed to control an intelligent crane system (Wahyudi and Jalani; 2005). Wang and Surgenor (2004) presented an optimal control method to control the dynamic motion of the system. They considered the minimum energy of system and integrated an absolute error of payload angle as their optimisation criterion.

This paper deals with the tracking control of an underactuated gantry crane employing an optimal feedback controller. At first, the nonlinear dynamic equations of the gantry crane are derived by the implementation of Lagrange's principle. Then, the linearised state-space equations of the system are presented, considering the motor voltage of a wheel of the trolley as the input, and the displacement of the trolley as the output of the crane. Considering the tracking response of the trolley and the minimum oscillation of the hoisting line as the optimisation criterions, an optimal feedback controller is designed based on the ITAE criterion. Finally, some simulation results are determined, which show the capability of the method for the optimal tracking control of a gantry crane.

\section{DYNAMIC MODEL OF SYSTEM}

In this section, the dynamic model of a gantry crane is presented. The dynamic equations of the system are derived using Lagrange's principle. Figure 1 shows a gantry crane moving in two-dimensional space. The crane consists of a cart (trolley) transversing in the horizontal direction, while a massless pendulum is connected to the cart and hoists the payload. The parameters of the overhead system are presented in Table 1. 


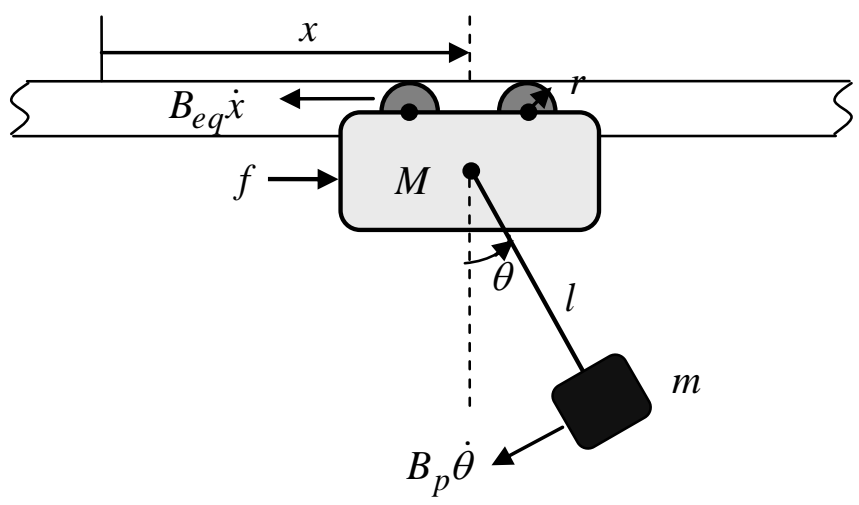

Figure 1. Gantry crane

Table 1. Parameters of the gantry crane

\begin{tabular}{lc}
\hline \multicolumn{1}{c}{ Parameters } & Nomenclature \\
\hline Cart position & $x$ \\
Cart velocity & $\dot{x}$ \\
Pendulum angular displacement & $\theta$ \\
Pendulum angular velocity & $\dot{\theta}$ \\
Pendulum length & $l$ \\
Mass of the cart system & $M$ \\
Payload mass & $m$ \\
Gravitational constant of earth & $g$ \\
Radius of wheels of cart & $r$ \\
DC motor voltage of cart & $e$ \\
Force exerted to cart & $f$ \\
Motor armature resistance & $R$ \\
Motor torque constant & $k$ \\
Viscous damping coefficient of pendulum & $B_{p}$ \\
axis & \\
Equivalent viscous damping coefficient & $B_{e q}$ \\
\hline
\end{tabular}

Hence equivalent viscous damping is presumed for cart displacement, and equivalent viscous damping is considered in the pendulum axis, thus the nonlinear dynamics of the gantry crane (shown in Fig. 1) can be summarised as (Dey et al., 2010):

$$
\begin{aligned}
& (M+m) \ddot{x}+m l\left(\ddot{\theta} \cos \theta-\dot{\theta}^{2} \sin \theta\right)+2 m \dot{l} \dot{\theta} \cos \theta+m \ddot{l} \sin \theta=f-B_{e q} \dot{x} \\
& l \ddot{\theta}+2 i \dot{\theta}+\ddot{x} \cos \theta+g \sin \theta=-B_{p} \dot{\theta}
\end{aligned}
$$

Moreover, the following simplifications can be applied to the system: (i) the model does not include a hoisting drive, thus the rod length is fixed; (ii) the trolley or payload is assumed to be point mass; (iii) the trolley and payload are assumed to move in the X-Y plane; and (iv) viscous damping effects are neglected. 
Applying the foregoing assumptions, the nonlinear dynamic equations of the system are presented in Eq. (2) (Ghosh et al., 2010):

$$
\begin{aligned}
& (M+m) \ddot{x}+m l \ddot{\theta} \cos \theta-m l \dot{\theta}^{2} \sin \theta=f \\
& \ddot{x} \cos \theta+l \ddot{\theta}+g \sin \theta=0
\end{aligned}
$$

In addition, the linear force $f$ originating from the torque of the motor of trolley $T$ (Friedland, 1986):

$$
\begin{aligned}
T & =r f \\
T & =\frac{k}{R} e-\frac{k^{2}}{R} \omega \\
\dot{x} & =r \omega
\end{aligned}
$$

where $\omega$ is the angular velocity of the DC motor and is related to the velocity of the cart by Eq. (3).

Furthermore, by combining Eq. (2) and Eq. (3), the nonlinear equation of the gantry cane considering an electrical motor can be summarised as follows:

$$
\begin{aligned}
& (M+m) \ddot{x}+m l \ddot{\theta} \cos \theta-m l \dot{\theta}^{2} \sin \theta=\frac{1}{r}\left(\frac{k}{R} e-\frac{k^{2}}{R r} \dot{x}\right) \\
& \ddot{x} \cos \theta+l \ddot{\theta}+g \sin \theta=0
\end{aligned}
$$

Furthermore, the state vector is defined as $\vec{X}=\left[\begin{array}{llll}x & \dot{x} & \theta & \dot{\theta}\end{array}\right]^{T}=\left[\begin{array}{llll}x_{1} & x_{2} & x_{3} & x_{4}\end{array}\right]^{T}$, and the nonlinear equations of the system in state-space form are presented as Eq. (5):

$$
\left\{\begin{array}{c}
\dot{x}=\frac{d}{d t}(x) \\
\ddot{x}=\frac{m l^{2} \dot{\theta}^{2} \sin \theta+(M+m) g l \tan \theta+l\left(\frac{k e}{R r}-\frac{k^{2} \dot{x}}{R r^{2}}\right)}{M+m\left(1-\cos ^{2} \theta\right)}-g \tan \theta \\
\dot{\theta}=\frac{d}{d t}(\theta) \\
\ddot{\theta}=\frac{-m l \dot{\theta}^{2} \sin \theta \cos \theta-(M+m) g \sin \theta-\cos \theta\left(\frac{k e}{R r}-\frac{k^{2} \dot{x}}{R r^{2}}\right)}{M l+m l\left(1-\cos ^{2} \theta\right)}
\end{array}\right.
$$

For safe operation, the swing angle should be kept small $(\theta \approx 0, \dot{\theta} \approx 0)$. Thus, the linearised state-space equations of the system can be presented as Eq. (6):

$$
\begin{aligned}
& \dot{\vec{X}}=A \vec{X}+B u \\
& y=C \vec{X}+D u
\end{aligned}
$$


where the matrices are:

$$
\begin{aligned}
A & =\left[\begin{array}{cccc}
0 & 1 & 0 & 0 \\
0 & -\frac{k^{2}}{R r^{2} M} & \frac{m g}{M} & 0 \\
0 & 0 & 0 & 1 \\
0 & \frac{k^{2}}{R r^{2} M l} & -\frac{(M+m) g}{M l} & 0
\end{array}\right] \quad B=\left[\begin{array}{c}
0 \\
\frac{k}{R r M} \\
0 \\
-\frac{k}{R r M l}
\end{array}\right] \\
C & =\left[\begin{array}{llll}
1 & 0 & 0 & 0
\end{array}\right]
\end{aligned}
$$

\section{Optimal Feedback Control Law}

In this section, the optimal feedback controller formulation is presented. Assuming that the system is described by Eq. (7), and the output $y=x_{1}$ must track the desired value $r$. Then the following state feedback control is defined (Ogata, 2002):

$$
u=-k_{1}\left(x_{1}-r\right)-\left[\begin{array}{lllll}
0 & k_{2} & k_{3} & \ldots & k_{n}
\end{array}\right] \vec{X}=+k_{1} r-\mathrm{K} \vec{X}
$$

where the feedback controller is $\mathrm{K}=\left[\begin{array}{lllll}k_{1} & k_{2} & k_{3} & \ldots & k_{n}\end{array}\right]$.

For optimal control of the system, the performance index is presumed as a function of integrated time and in absolute error terms:

$$
J_{\text {ITAE }}=\int_{t_{0}}^{t_{f}} t|e(t)| d t
$$

Hence the cost function is defined as Eq. (9), the characteristic equation of the closed-loop system can be obtained as an ITAE polynomial (Preitl et al., 2008):

$$
\Delta_{\text {ITAE }}(s)=s^{4}+2.1 \omega_{n} s^{3}+3.4 \omega_{n}^{2} s^{2}+2.7 \omega_{n}^{3} s+\omega_{n}^{4}
$$

where $\omega_{n}$ is a dimensionless parameter and can be changed by the designer to achieve the best response from the closed-loop system.

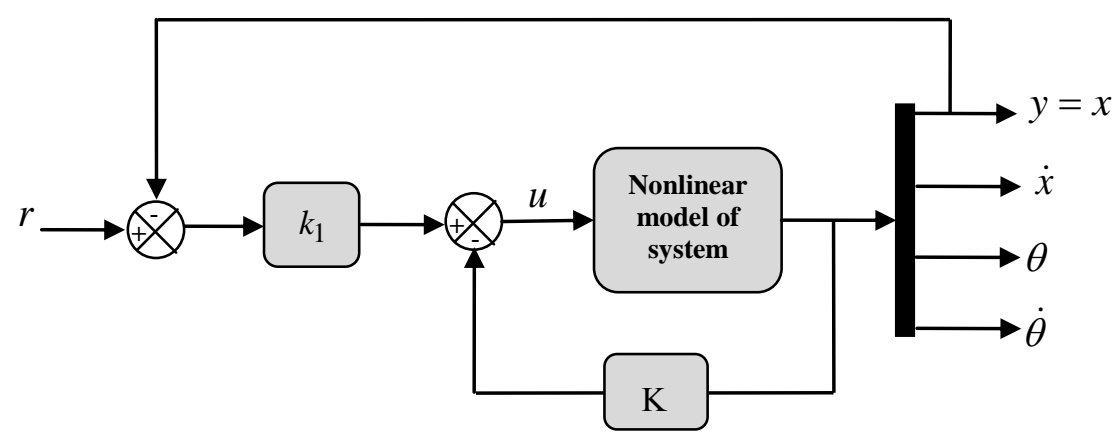

Figure 2. Scheme of feedback controller 


\section{RESULTS AND DISCUSSION}

In this section the tracking control of the gantry crane is simulated. For the gantry crane presented (Figure 1), it is desired that the cart tracks a step value in minimum time, while the oscillation of the pendulum is quickly settled. The parameter values of the system are presented in Table 2 .

Table 2. Parameter values of the gantry crane (Iles et al., 2011)

\begin{tabular}{lcc}
\hline \multicolumn{1}{c}{ Parameter } & Value & Unit \\
\hline Pendulum length $(l)$ & 0.3302 & $\mathrm{~m}$ \\
Mass of the cart system $(M)$ & 1.073 & $\mathrm{~kg}$ \\
Payload mass $(m)$ & 0.23 & $\mathrm{~kg}$ \\
Gravitational constant of earth $(g)$ & 9.81 & $\mathrm{~m} / \mathrm{s}^{2}$ \\
Radius of wheels of cart $(r)$ & 0.006 & $\mathrm{~m}$ \\
Motor armature resistance $(R)$ & 2.6 & $\Omega$ \\
Motor maximum voltage $\left(e_{\max }\right)$ & 12 & $\mathrm{~V}$ \\
Motor torque constant $(k)$ & 0.00767 & $\mathrm{Vs} / \mathrm{rad}$ \\
\hline
\end{tabular}

As mentioned, the desired criterion of the control design are: the trolley can track the reference input while the sway of the pendulum is quickly dampened, and the input voltage of the motor does not exceed its maximum value. It must be noted that the simulations are performed in Simulink of MATLAB. Hence, the control law is developed for the linear form of the system, but the nonlinear model is simulated in the studies. To achieve the best parameters of design, the value of $\omega_{n}$ in Eq. (10) is changed, and the corresponding responses are evaluated. Then, the best value of $\omega_{n}$ is chosen, and the response of the system and input voltage are determined. Assuming different values of $\omega_{n}$, the desired poles of the closed-loop system, and the gain matrix $K$ are presented in Table 3.

Table 3. Closed-loop poles of the system

\begin{tabular}{cccccc}
\hline$w_{n}$ & Desired closed-loop poles & \multicolumn{5}{c}{ Gain matrix K } \\
\hline 1 & $P_{1,2}=-0.626 \pm 0.414 i$ & $K=\left[\begin{array}{lllll}0.073458 & -1.08 & 23.572 & -1.447\end{array}\right]$ \\
& $P_{3,4}=-0.424 \pm 1.263 i$ \\
2 & $P_{1,2}=-0.6848 \pm 2.526 i$ & $K=\left[\begin{array}{lllll}1.1753 & 0.30835 & 16.586 & -2.5027\end{array}\right]$ \\
& $P_{3,4}=-1.252 \pm 0.828 i$ \\
3 & $P_{1,2}=-1.2719 \pm 3.789 i$, & $K=\left[\begin{array}{lllll}5.9501 & 4.0767 & 5.9119 & -2.7717\end{array}\right]$ \\
& $P_{3,4}=-1.8781 \pm 1.2424 i$ & & & & \\
4 & $P_{1,2}=-1.6959 \pm 5.052 i$ & $K=\left[\begin{array}{lllll}18.805 & 11.415 & -6.9941 & -1.8618\end{array}\right]$ \\
& $P_{3,4}=-2.5041 \pm 01.6566 i$ & & & & \\
\hline
\end{tabular}

As the input reference is assumed to be a unit step, the trolley displacement of the gantry crane is shown in Figure 3. The angular displacement of the pendulum is shown in Figure 4. Moreover, the input voltage of the cart of the gantry crane during 
motion is shown in Figure 5. As is seen in Figures 3-5, the cart and pendulum displacements, and the input voltage are depicted against the time of motion.

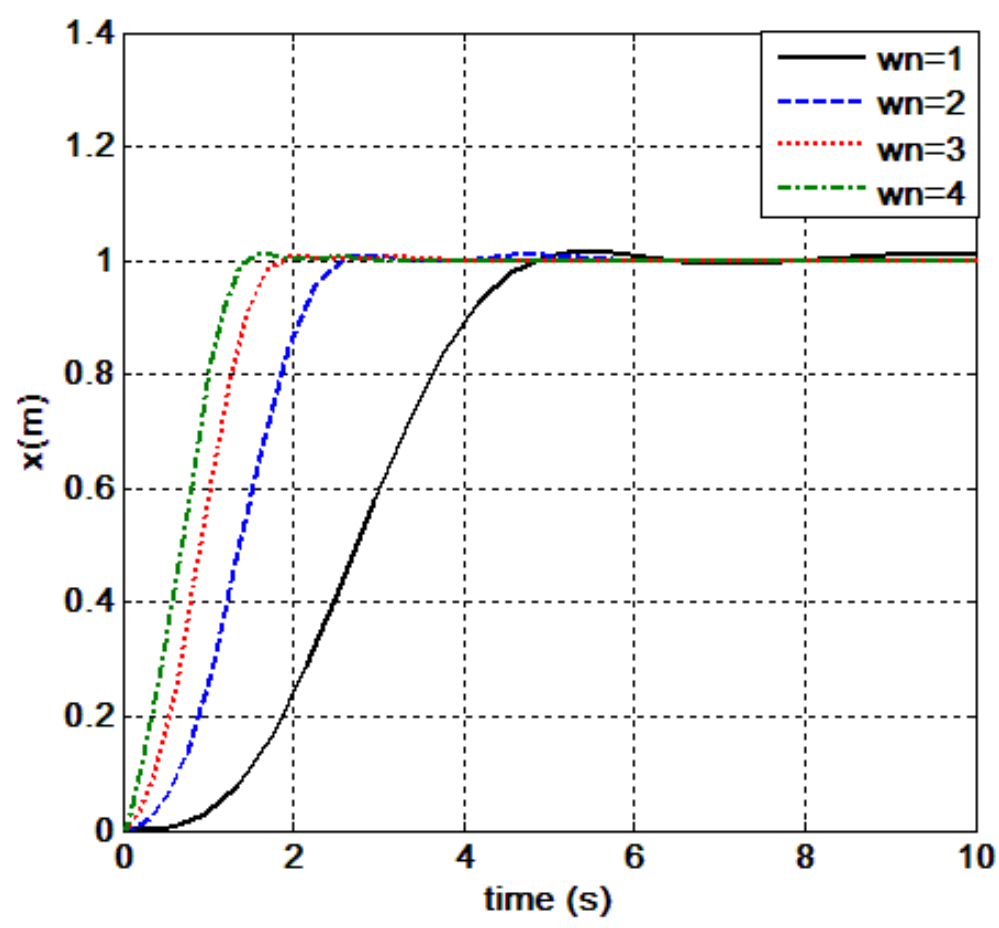

Figure 3. Displacement of the cart

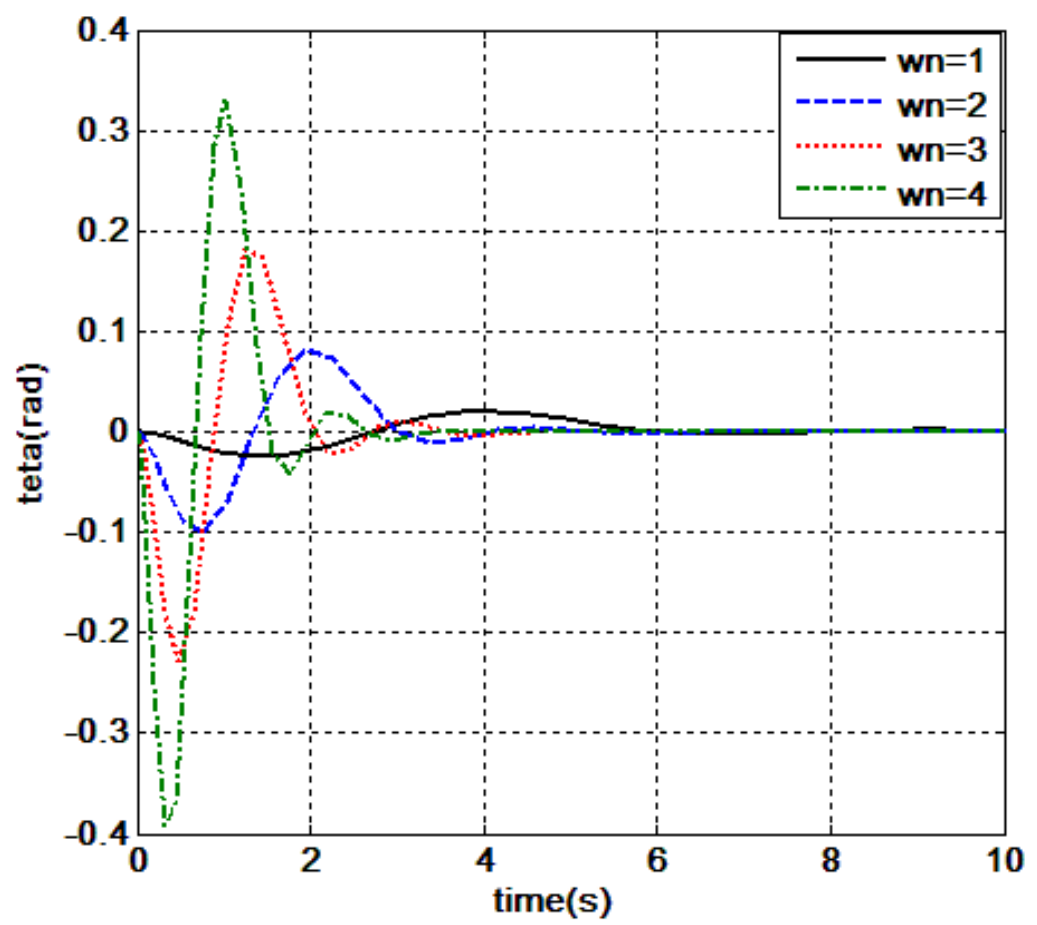

Figure 4. Angular displacement of the pendulum 


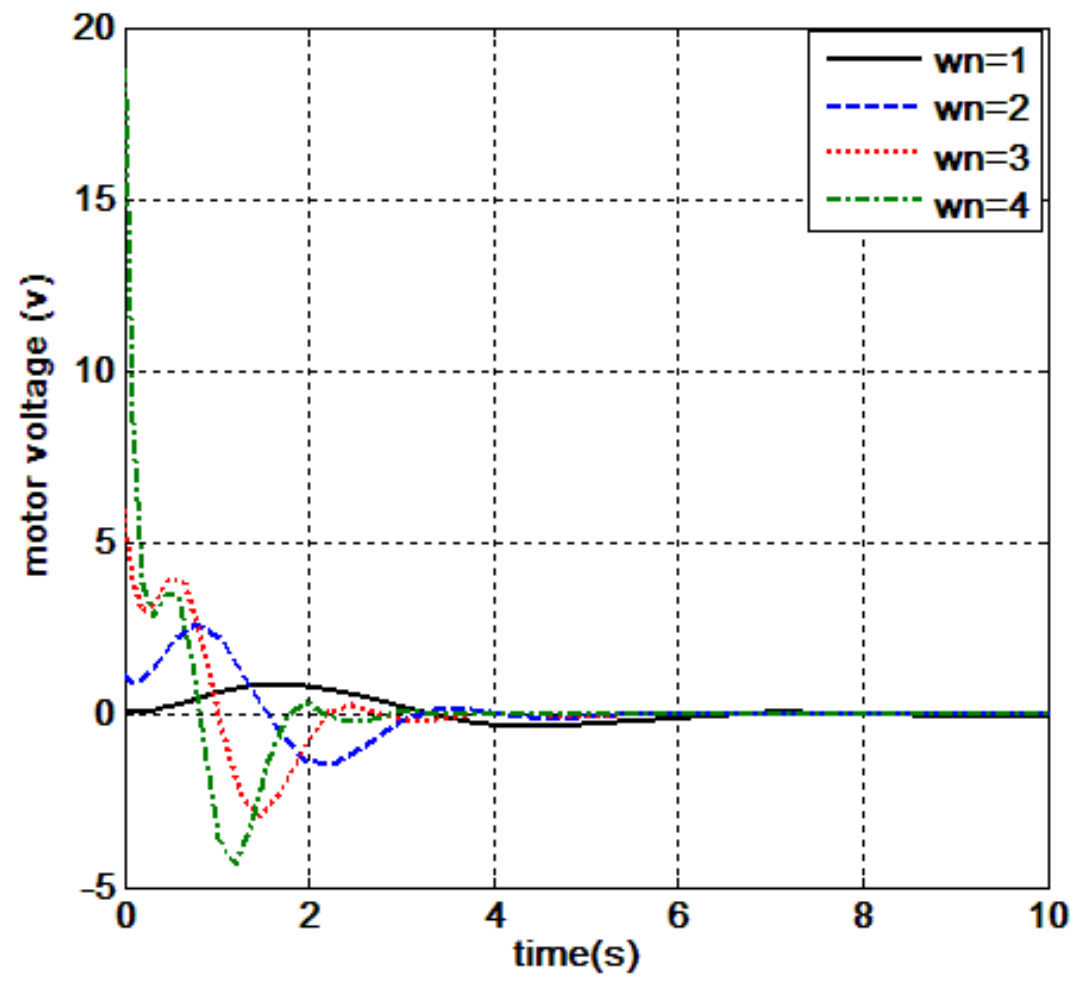

Figure 5. Motor voltage of the cart

As seen in Figure 3, by increasing the value of $\omega_{n}$ the tracking error of the cart displacement decreases, and the response to the reference input becomes more rapid. But according to Figures 4 and 5, increasing the value of $\omega_{n}$ results in an increase in the maximum values of the angular displacement of the pendulum and the motor voltage of the cart. This matter stems from the fact that increasing the gains of the optimal feedback controller results in exceeding of the input efforts of the system. Thus the designer must perform a trade-off between the criterion, according to the characteristics of the response, such as the settling time and maximum overshoot of the cart displacement, the maximum value of the angular displacement of the pendulum, and the maximum value of the motor voltage. Table 4 shows the characteristics of the system's response when considering different values of $\omega_{n}$.

Table 4. Characteristics of response of the system

\begin{tabular}{cccccc}
\hline$w_{n}$ & $\begin{array}{c}\text { Maximum } \\
\text { percent } \\
\text { overshoot of } x\end{array}$ & $\begin{array}{c}\text { Settling time } \\
\text { of } x\end{array}$ & $\begin{array}{c}\text { Maximum } \\
\text { value of } \theta\end{array}$ & $\begin{array}{c}\text { Settling } \\
\text { time of } \theta\end{array}$ & $\begin{array}{c}\text { Maximum } \\
\text { voltage of } \\
\text { motor }\end{array}$ \\
\hline 1 & 0.6711 & 4.155 & 0.0251 & 6.1550 & 0.90895 \\
2 & 0.97146 & 1.9383 & 0.1002 & 4.7701 & 2.6317 \\
3 & 0.7819 & 1.4622 & 0.2273 & 4.5551 & 5.9501 \\
4 & 1.0937 & 1.1934 & 0.3930 & 3.6678 & 18.805 \\
\hline
\end{tabular}

According to Table 4, as the value of $\omega_{n}$ increases the settling time of $x, \theta$ is decreases, which is a criterion of the design. But the maximum values of the angular 
displacement of the pendulum and the input voltage of the motor are increased, which are not appropriate tasks for the design of the controller. Thus, based on the ITAE criterion, the best closed-loop poles are obtained when $\omega_{n}=3$, and is equal to $P_{1,2}=-1.2719 \pm 3.789 i, P_{3,4}=-1.8781 \pm 1.2424 i$ and the corresponding gain matrix is $K=\left[\begin{array}{llll}5.9501 & 4.0767 & 5.9119 & -2.7717\end{array}\right]$. Thus the gain matrix of the feedback controller is able to trade between all the criterion of the design, such as a fast response of the cart, minimum swing angle, and reasonable values for input effort of the system without any saturation.

\section{CONCLUSION}

In the presented paper, an optimal feedback controller has been designed for a gantry crane, which can track a desired reference input, taking into account ITAE criterion. The nonlinear dynamic equations of the system have been derived, and the nonlinear and linearised state-space dynamic models of the gantry crane have been presented. Moreover, a scheme of the feedback control law to track a reference value has been developed, and the desired closed-loop characteristic equation of the system has been obtained by employing the ITAE polynomial. Then, some simulations of the system have been presented using different values of the closed-loop system. The desired criterion of the control design have been defined in that the trolley must track the reference input while the swing angle of the pendulum is dampened quickly, and the input voltage of the motor does not exceed its maximum value. Therefore, the best gain matrix of the feedback controller has been obtained. It has been shown that the proposed method is simple and effective for the optimal tracking of a desired input.

\section{REFERENCES}

Abdel-Rahman, E.M., Nayfeh, A.H. and Masoud, Z.N. 2003. Dynamic and control of cranes: a review. Journal of Vibration and Control, 9: 863-908.

Ahmad, M.A. 2009. Active sway suppression techniques of a gantry crane system. European Journal of Scientific Research, 27: 322-333.

Ahmad, M.A. and Mohamed Z. 2009. Hybrid fuzzy logic control with input shaping for input tracking and sway suppression of a gantry crane system. American Journal of Engineering and Applied Sciences, 2: 241-251.

Ahmad, M.A., Nasir, A.N.K., Hambali, N. and Ishak, H. 2009. Hybrid input shaping and PD-type fuzzy logic control scheme of a gantry crane system. International Conference on Control Applications, Russia, pp. 1051-1056.

Ahmad, M.A., Nasir, A.N.K., Raja Ismail, R.M.T. and Ramli, M.S. 2010. Control schemes for input tracking and anti-sway control of a gantry crane. Australian Journal of Basic and Applied Sciences, 4: 2280-2291.

Dey, R., Sinha, N., Chaube, P., Ghosh, S. and Ray, G. 2010. Active sway control of a single pendulum gantry crane system using output-delayed feedback control technique. International Conference on Control, Automation, Robotics and Vision, pp. 532-536.

Friedland, B. 1986. Control system design. USA: McGraw-Hill.

Ghosh, S., Ray, G., Dey, R., Sinha, N. and Chaubey, P. 2010. Active sway control of a single pendulum gantry crane system using output-delayed feedback control 
technique. International Conference on Control, Automation, Robotics and Vision, pp. 532-536.

Hazlerigg, A.D.G. 1972. Automatic control of crane operations. Proceedings of IFAC World Congress, pp. 32-38.

Hubbell, J.T., Koch, B. and McCormick, D. 1992. Modern crane control enhancements. Seattle: ASCE.

Iles, S., Koloni, F. and Matusko, J. 2011. Linear matrix inequalities based H1 control of gantry crane using tensor product transformation. International Conference on Process Control, pp. 92-99.

Kolonic, F., Poljugan, A. and Petrovic, I. 2006. Tensor product model transformationbased controller design for gantry crane control system - an application approach. Journal of Acta Polytechnica Hungarica, 3: 95-112.

Manning, R., Clement, J., Kim, D. and Singhose, W. 2010. Dynamics and control of bridge cranes transporting distributed-mass payloads. Journal of Dynamics Systems, Measurement, and Control, 132: 134-142.

Masoud, Z.N. 2009. Effect of hoisting cable elasticity on anti-sway controllers of quayside container cranes. Journal of Nonlinear Dynamics, 58: 129-140.

Masoud, Z.N. and Daqaq, M.F. 2006. A graphical approach to input-shaping control design for container cranes with hoist. Transactions on Control Systems Technology, 14: 1070-1076.

Moustafa, K.A.F. and Ebeid, A.M. 1988. Nonlinear modeling and control of an overhead crane load sway. ASME Transactions on Dynamics Systems, Measurement and Control, 110: 266-271.

Ogata, K. 2002. Modern control engineering. Upper Saddle River, NJ: Aeeizb Publishers.

Preitl, S., Precup, R.E., Clep, P.A., Ursache, I.B., Fodor, J. and Škrjanc, I. 2008. Pole placement approaches for linear and fuzzy systems. IEEE Conference on Control, pp. 213-219.

Rahn, C.D., Zhang, F., Joshi, S. and Dawson, D.M. 1999. Asymptotically stabilizing angle feedback for a flexible cable gantry crane. Journal of Dynamics Systems, Measurement, and Control, 121: 563-566.

Solihin, M.I. and Legowo, A. 2010. Fuzzy-tuned PID anti-swing control of automatic gantry crane. Journal of Vibration and Control, 16: 127-145.

Stahlbock, R. and Vob, S. 2010. Efficiency considerations for sequencing and scheduling of double-rail-mounted gantry cranes at maritime container terminals. International Journal of Shipping and Transport Logistics, 2: 95-123.

Wahyudi, M. and Jalani, J. 2005. Design and implementation of fuzzy logic controller for an intelligent gantry crane system. Proceedings of International Conference on Mechatronics, pp. 345- 351.

Wang, Z. and Surgenor, B. 2004. Performance evaluation of the optimal control of a gantry crane. ASME Conference on Engineering Systems Design and Analysis, pp. 869-874.

Yu, J., Lewis, F.L. and Huang, T. 1995. Nonlinear feedback control of a gantry crane. Proceedings of American Control Conference, pp. 4310-4315.

Zrnic, N.D., Petcovic, Z.D., Ostric, D.Z. and Brkic. A.D. 1998. On a method for defining horizontal of gantry cranes. Proceedings of Conference on Manufacturing Engineering, pp. 573-580. 